\title{
Interpersonal violence as a major contributor towards the skewed burden of trauma in KwaZulu-Natal, South Africa
}

\author{
C Lewis, MB ChB, Dip ROM RCSEd; D Wood, MB BCh, FCEM, MPhil, BPharm \\ Department of Emergency Medicine, School of Clinical Medicine, College of Health Sciences, Nelson R Mandela School of Medicine, \\ University of KwaZulu-Natal, Durban, South Africa
}

Corresponding author: C Lewis (drcarolynlewis@yahoo.com)

Background. Trauma is one of the foremost causes of death worldwide, but there is a paucity of data on demographics and injury patterns in developing countries.

Objectives. To quantify and describe the major trauma burden at a regional hospital in KwaZulu-Natal (KZN), South Africa (SA), over a 5 -year period.

Methods. Ngwelezane Hospital is a large regional hospital in northern KZN. A database is maintained of all major trauma patients admitted to the Emergency Department (ED) resuscitation unit. Statistical analysis was performed to quantify the burden of trauma and analyse trends in these data.

Results. Over a 5-year period, 3735 major trauma patients were admitted to the ED resuscitation unit. Analysis showed a male predominance, with a mean patient age of 28.6 years. An average of 62 patients per month were admitted, the rate peaking over the holiday seasons. Trauma secondary to interpersonal violence (IPV) predominated. A disproportionately high level of pedestrian-motor vehicle collisions (PMVCs) in relation to total road traffic collisions was noted. Blunt force trauma secondary to motor vehicle collisions was the leading cause of death, while blunt force trauma secondary to PMVCs carried the highest mortality rate.

Conclusion. This study highlights the high incidences of both IPV and PMVCs typical of trauma in an SA setting. The demographics and injury patterns noted may be used to drive public health interventions to address this burden of trauma.

S Afr Med J 2015;105(10):827-830. DOI:10.7196/SAMJnew.8380

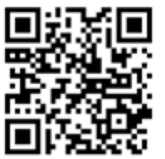

Trauma is a leading cause of death worldwide, with a higher incidence in low- and middle-income countries than in high-income countries. ${ }^{[1]}$ There is a paucity of data on demographics and injury patterns in developing countries, and without such data public health interventions to address this burden cannot be accomplished. ${ }^{[2]}$

Muckart ${ }^{[3]}$ likened trauma in South Africa (SA) to a malignant epidemic almost 25 years ago. Little has changed since then, and SA has maintained its place as one of the 'capitals' of trauma in the world. What is of concern is the type of trauma being reported, i.e. the high incidences of interpersonal violence (IPV) and pedestrian-motor vehicle collisions (PMVCs). KwaZulu-Natal Province (KZN) is the most populous of SA's nine provinces, with almost 11 million people. ${ }^{[4]}$ Numerous studies have analysed various aspects of trauma in KZN. Hardcastle et al. ${ }^{[5]}$ assessed the burden of trauma with regard to care provided in public hospitals over a 2-month period, and showed a high burden of trauma with a predominance of intentional trauma. Parkinson et al. ${ }^{[6]}$ assessed the burden of trauma secondary to road traffic collisions (RTCs) in a regional trauma centre in KZN over a 10-week period, their data highlighting both a significant burden of trauma secondary to RTCs and a relatively high proportion of PMVCs. Laing et al. ${ }^{[7]}$ assessed the burden of trauma in the Pietermaritzburg Metropolitan Trauma Service throughout 2013. These studies all demonstrated a high incidence of trauma and alluded to the skewed number of IPV cases with significant morbidity and mortality outcomes. We aimed to add to the reported data by quantifying the trauma burden at a large regional hospital in KZN over an extended period and sought to evaluate trends from the extracted data.

\section{Objectives}

To describe the incidence rate of major trauma at a regional hospital in KZN with a focus on the demographics of trauma patients, seasonal trends, mechanism of injury, length of stay, disposition and mortality.

\section{Methods}

We analysed data from Ngwelezane Hospital (NH), a 554-bed regional hospital in northeastern KZN, over the 5-year period from January 2009 - December 2013. All major trauma in the region is referred to $\mathrm{NH}$ through the hospital's emergency department (ED). The resuscitation unit of the $\mathrm{ED}$ at $\mathrm{NH}$ is responsible for assessment and admission of all patients with major injuries. All patients are triaged on arrival. Major injuries are defined according to the South African Triage Scale (SATS) as red (physiologically unstable patients) or orange (serious cases with potentially unstable physiology or potentially life-threatening or limb-threatening pathology). ${ }^{[8]}$

The ED maintains a Microsoft Office Access database (based on MS Excel 2007) of all patients admitted to its resuscitation unit. Data are collected prospectively on all patients admitted to the ED by the senior medical officer or specialist trainee on call during the 24-hour on-call period.

The study population included all persons within the drainage area of $\mathrm{NH}$. The sampling frame was all patients admitted to the resuscitation unit at NH from January 2009 to December 2013, inclusive. The study sample was the subset of these patients whose admissions were a result of major trauma. Patients with minor trauma who did not require admission to the resuscitation unit for stabilisation, and non-trauma-related admissions, were excluded from analysis.

Data extracted included age, gender, injury type, month of admission, length of hospital stay, disposition and mortality. Statistical analysis was performed using Intercooled Stata version 13 


\section{Ethical considerations}

Ethics approval was obtained from the University of KwaZulu-Natal Biomedical Research Ethics Committee (BE022/14).

\section{Results}

A total of 10390 patients were seen as emergencies during the period January 2009 - December 2013. Of these 3735 were classified as having sustained major trauma, and their demographics were as follows: 3036 (81.3\%) were male, 671 (18.1\%) were female, and 28 had no gender documented. The mean age was 28.6 years (standard deviation 13.55). The majority of patients admitted were between 20 and 30 years of age (Fig. 1). An average of 62 patients a month were admitted, with the rate peaking over the months of January, April and May (Fig. 2).

Breakdown of all cases of major trauma showed the following: IPV 2594 patients (69.4\%), with penetrating trauma secondary to stabbing in $911(24.3 \%)$, isolated head injury in $632(16.9 \%)$, penetrating trauma secondary to gunshot wounds (GSWs) in $542(14.5 \%)$, crush injury secondary to community assault in 340 (9.1\%), blunt force trauma secondary to assault in 170 (4.5\%), blunt force trauma secondary to motor vehicle collisions (MVCs) in 650 (17.4\%), blunt force trauma secondary to PMVCs in 285 (7.6\%), and burns in 205 (5.4\%). Our experience is that most isolated head injuries are caused by IPV. Fig. 3 summarises the mechanisms of injury.

One hundred and sixty-eight patients died while admitted in the resuscitation unit of the $\mathrm{ED}$, giving a mortality rate of $4.5 \%$ and a case fatality rate of 1.6/100 admissions. Analysis of the mechanism of injury resulting in death revealed that IPV predominated, with 92 deaths (54.7\%). Of the patients who died, $39(23.2 \%)$ sustained penetrating trauma secondary to GSWs, $36(21.4 \%)$ an isolated head injury, 7 (4.1\%) penetrating trauma secondary to stabbing, 7 (4.1\%) blunt force trauma assault, and $3(1.7 \%)$ crush injury secondary to community assault. Other causes of death were as follows: 42 patients (25.0\%) sustained blunt force trauma secondary to an MVC, 25 (14.8\%) blunt force trauma secondary to a PMVC, and 9 (5.3\%) burns. Fig. 4 summarises mortality according to mechanism of injury.

The single mechanism of injury responsible for the highest overall mortality was blunt force trauma secondary to a PMVC (8.7\%), followed by penetrating trauma secondary to a GSW (7.1\%) and blunt force trauma secondary to an MVC (6.4\%). These findings are summarised in Table 1.

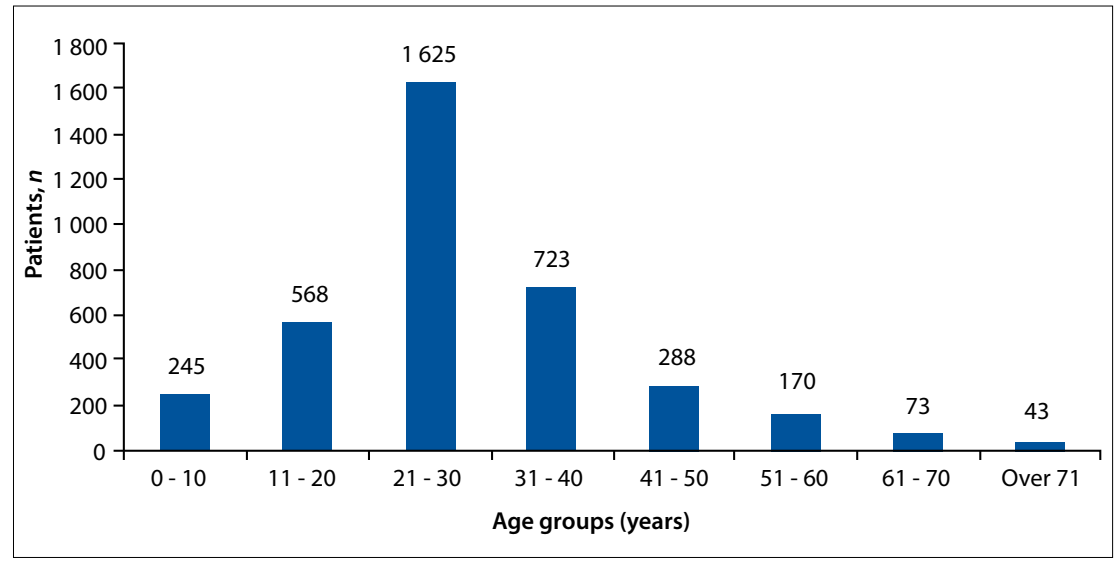

Fig. 1. Breakdown of patients according to age group.

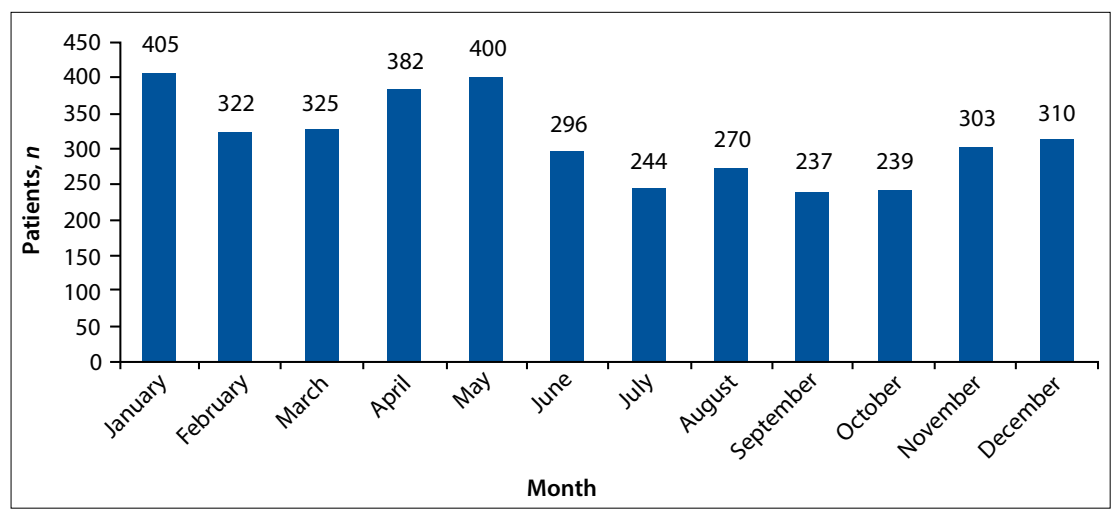

Fig. 2. Monthly admissions over a 5-year period.

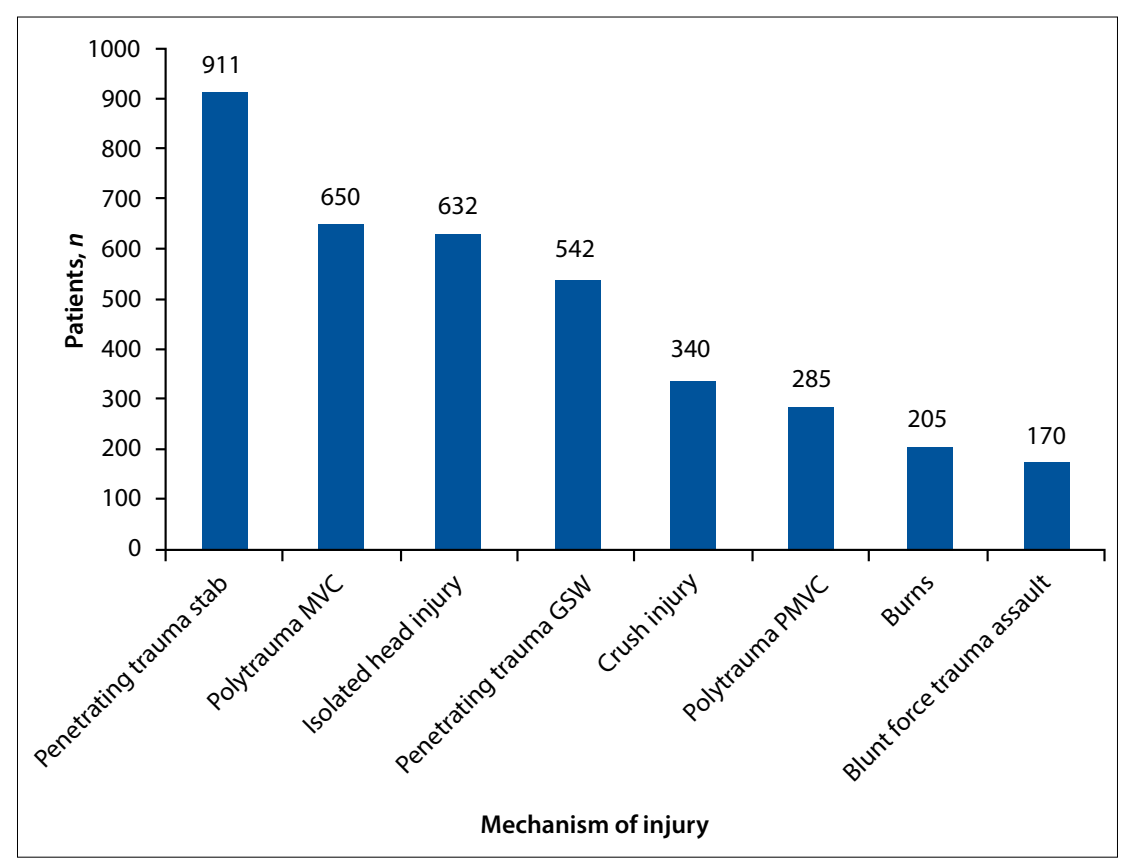

Fig. 3. Mechanisms of injury.

The majority of patients ( $n=2717$ ) admitted to the resuscitation unit were admitted for $<12$ hours $(72.7 \%)$. Of those admitted, 1942 patients (51.9\%) were discharged from the resuscitation unit ward of the ED to a general ward, 715 (19.1\%) went to theatre directly from the unit, 379 (10.1\%) were discharged home once stabilised, 257 (6.8\%) were discharged to a peripheral hospital, 248 $(6.6 \%)$ were transferred to an intensive care unit, and $26(0.6 \%)$ were transferred to a private hospital. 


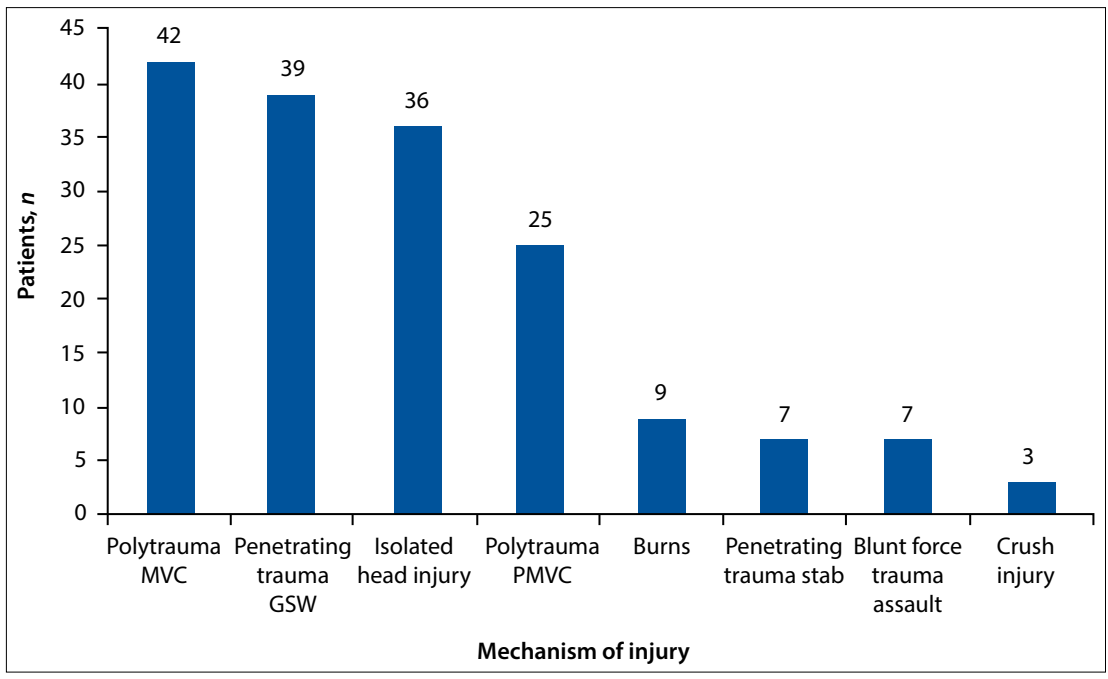

Fig. 4. Mechanisms of injury causing death.

Table 1. Mechanism of injury v. mortality

\begin{tabular}{lllll}
\hline & $\begin{array}{l}\text { Mechanism } \\
\text { of injury }\end{array}$ & Deaths & $\begin{array}{l}\text { Mortality } \\
\text { rate (\%) }\end{array}$ & $\begin{array}{l}\text { Case fatality } \\
\text { rate/100 } \\
\text { admissions }\end{array}$ \\
\hline Polytrauma PMVC & 285 & 25 & 8.7 & 0.2 \\
Penetrating trauma GSW & 542 & 39 & 7.1 & 0.3 \\
Polytrauma MVC & 650 & 42 & 6.4 & 0.4 \\
Isolated head injury & 632 & 36 & 5.6 & 0.3 \\
Burns & 205 & 9 & 4.3 & 0.08 \\
Blunt force trauma assault & 170 & 7 & 4.1 & 0.06 \\
Crush injury & 340 & 3 & 0.8 & 0.02 \\
Penetrating trauma stab & 911 & 7 & 0.8 & 0.06
\end{tabular}

\section{Discussion}

Hospitals in developing countries manage a large volume and range of major trauma. In contrast to the spectrum of trauma seen in a developed world, where blunt force trauma secondary to RTCs and self-inflicted injuries predominates, ${ }^{[1]}$ a significant proportion of major trauma admissions to $\mathrm{NH}$ are the result of IPV. Hofman et al. ${ }^{[2]}$ describe the mortality rate for injury secondary to intentional violence as ten times higher in low- and middle-income countries than in high-income countries. Similar patterns are observed for disability-adjusted life-years. This observation is borne out in our study.

The demographic group most affected by trauma was males aged 20 - 30 years, in whom the majority of injuries are due to IPV. There was a peak in admission rates over the months of January, April and May. This peak distribution was consistent over the study period, but unexpectedly did not appear to be associated with all the holiday seasons throughout the year. There was a trauma caseload peak over the
Easter April/May season, but the Christmas and New Year holidays did not show a significant peak in trauma cases. While our results do not take specific days into account, our experience is that there are significant trauma peaks on holidays such as Christmas Day, the Day of Goodwill (Boxing Day) and New Year's Day.

Penetrating trauma secondary to stabs and GSWs were significant contributors to the trauma numbers in our study. The high proportion of crush injuries following assault is also striking. Crush syndrome following assault is a uniquely SA phenomenon ${ }^{[9]}$ and is usually the result of community justice or 'kangaroo courts'. secondary to muscle trauma with the risk of acute kidney injury. Many of these patients also suffer defence injuries such as fractures of the forearm.

The high rate of IPV also contributes to the number of isolated head injuries. The incidence rate for isolated severe head injuries in our study is $24 / 100000$ population These patients sustain rhabdomyolysis in our district. International and currently available SA data assess all traumatic brain injuries (TBIs), mild, moderate and severe, of which mild TBIs constitute 75 - 95\%. ${ }^{[10,11]}$ Our findings reflect those of the World Health Organization (WHO), which demonstrated an increased incidence rate of all TBIs from 150 to 170/100 000 in Latin America and sub-Saharan Africa, respectively, in contrast to the global rate of 106/100 000. ${ }^{[12]}$ Over two decades ago, Nell and Brown ${ }^{[13]}$ documented an annual incidence rate of TBIs (mild, moderate and severe) of 316/100 000 in Johannesburg, SA. International data suggest that nearly $60 \%$ of all traumatic head injuries are a result of RTCs, $20-30 \%$ are due to falls, $10 \%$ are due to IPV, and another $10 \%$ are a result of a combination of workplace and sports-related injuries, ${ }^{[12]}$ whereas the high SA incidence rates reflect the high levels of IPV typical of SA trauma. ${ }^{[13]}$ Despite their different study population, Hardcastle et $a l .{ }^{[5]}$ also found a high incidence of IPV.

Parkinson et al. ${ }^{[6]}$ assessed the burden of victims of RTCs presenting to Edendale Hospital (one of the major trauma centres in Pietermaritzburg, the second-largest city in KZN). They demonstrated a high burden of trauma as a result of RTCs, with PMVCs accounting for $41 \%$. Of 935 admissions secondary to RTCs in our study, $69.5 \%$ were due to MVCs and $30.5 \%$ to PMVCs. Based on estimated global road traffic fatalities, PMVCs account for $22 \%$ of all road traffic deaths worldwide. ${ }^{[14]}$ The WHO notes a clear geographical distribution, with the proportion of pedestrians killed in relation to other road users highest in the African region (38\%). ${ }^{[14]}$ Both Parkinson et al'.s ${ }^{[6]}$ and our study correlate with the findings of the WHO.

IPV among young adult males is a major contributor to the trauma burden in our study. The WHO notes that countries around the world are at differing stages with regard to their capacity for data collection. Although mortality data are widely collected all over the world, data pertaining to nonfatal outcomes are not available in most countries of the world. ${ }^{[1]}$ In comparison with WHO available data, the contribution of IPV to the total trauma burden is significantly higher than figures quoted in developed countries. ${ }^{[1]}$ In our experience, alcohol intake and lack of employment opportunities in the region are factors contributing to the reported violence. Studies are needed to identify factors that either increase or decrease the risk for violence and furthermore identify which of these factors can be modified through intervention. 
In our experience, the low socioeconomic status of our patient population often results in lack of transport and therefore a high proportion of pedestrians. Studies are needed to identify factors that increase or decrease the risk of RTCs, particularly PMVCs, in order to successfully implement effective interventions and legislation.

\section{Study limitations}

There are limitations to our study. Patients with major trauma were assessed according to the SATS (which is the standard triage of all emergency departments in SA) as red or orange, but no formal injury severity score or similar assessment was performed. Notably, a SATS score of red or orange does not necessarily correlate with injury severity; a physiologically unstable patient may rapidly become physiologically stable after treatment of their underlying condition (such as correction of hypovolaemic shock), which may account for many of the $10 \%$ of victims of major trauma in this study who were discharged directly home from the ED. While data were collected on all patients admitted to the ED prospectively, it is a limitation of our study that data may not have been accurately recorded on those holidays that anecdotally have peaks of trauma admissions, owing to the high workload and ensuing time constraints. This may result in the peak distribution of admission rates per month not reflecting all holiday seasons throughout the year.

\section{Conclusions}

Regional hospitals in developing countries attend to a large volume and wide variety of major trauma. Our study highlights a disproportionately high level of IPV with resultant morbidity and mortality when compared with developed countries. It further highlights the burden of PMVCs as a proportion of all RTCs. This high incidence of IPV and comparatively high incidence of PMVCs are areas that would benefit from further study, effective legislation and community intervention.
Acknowledgements. The authors wish to acknowledge that the design and maintenance of the ED Microsoft Office Access database from which data were collated for this study is attributed to Dr Naas Postma, MB ChB, DipEC, MPhil, of the Department of Emergency Medicine, University of KwaZulu-Natal.

\section{References}

1. Peden M, McGee K, Krug E. Injury - a Leading Cause of the Global Burden of Disease 2000. Geneva: World Health Organization, 2000

2. Hofman K, Primack A, Keusch, G, et al. Addressing the growing burden of trauma and injury in low- and middle-income countries. Am J Public Health 2005;95(1):13-17. [http://dx.doi.org/10.2105/ AJPH.2004.039354]

3. Muckart. Trauma - the malignant epidemic. S Afr Med J 1991;79(2):93-95.

4. Statistics South Africa. Census 2011. www.statssa.gov.za/publications/p03014/p030142011.pdf (accessed 22 December 2014).

5. Hardcastle T, Samuels C, Muckart D. An assessment of the hospital disease burden and the facilities for the in-hospital care of trauma in KwaZulu-Natal, South Africa. World J Surg 2013;37(7):1550-1561.
f [http://dx.doi.org/10.1007/s00268-012-1889-1]

6. Parkinson F, Kent S, Aldous C, et al. Road traffic crashes in South Africa: The burden of injury to a regional trauma centre. S Afr Med J 2013;103(11):850-852. [http://dx.doi.org/10.7196/ samj.6914]

7. Laing G, Skinner D, Bruce J, et al. Understanding the burden and outcome of trauma care drives a new trauma systems model. World J Surg 2014;38(7):1699-1706 [http://dx.doi.org/10.1007/s00268014-2448-8]

8. Gottschalk SB, Wood D, de Vries S, et al. The Cape Triage Score: A new triage system South Africa. Proposal from the Cape Triage Group. Emerg Med J 2006;23(2):149-153. [http://dx.doi.org/10.1136/ emj.2005.028332]

9. Muckart D, Abdool-Carrim A. Pigment-induced nephropathy after sjambok injuries. S Afr J Surg 1991;29(1):21-24.

10. Get the stats on traumatic brain injuries in the United States. 2013. www.cdc.gov/traumaticbraininjury (accessed 27 December 2014).

11. Evans R. Concussion and mild traumatic brain injury. 2014 UpToDate. www.uptodate.com (accessed 27 December 2014).

2. Hyder A, Wunderlich C, Puvanachandra P, et al. The impact of traumatic brain injuries: A global Hyder A, Wunderlich C, Puvanachandra P, et al. The
perspective. Neurorehabilitation 2007;22(5):341-353.

13. Nell V, Brown D. Epidemiology of traumatic brain injury in Johannesburg - II. Morbidity, mortality and etiology. Soc Sci Med 1991;33(3):289-296. [http://dx.doi.org/10.1016/0277-9536(91)90363-H]

14. World Health Organization. Pedestrian Safety: A Road Safety Manual for Decision Makers and Practitioners. 2013. www.who.int/roadsafety/en/ (accessed 12 February 2015).

15. Garrib A, Herbst A, Hosegood V, et al. Injury mortality in rural South Africa 2000-2007: Rates and associated factors. Trop Med Int Health 2011;16(4):439-446. [http://dx.doi.org/10.1111/j.13653156.2011.02730.x]

Accepted 16 July 2015 